\title{
Can we modify the Mediterranean diet for non-Mediterranean populations? Results from two randomised controlled trials.
}

\section{Abstract}

The Mediterranean diet is rich in bioactive nutrients and may be effective at preventing cardiovascular disease and dementia. However, long-term sustainability could be limited in non-Mediterranean populations with different nutrient requirements and food preferences. To address this issue, our research group conducted two randomised controlled trials to examine whether the Mediterranean diet can be adapted to increase sustainability for an Australian population, while still providing cardiovascular and cognitive benefits. In our first trial $(n=41)$, we examined a Mediterranean diet designed to meet the calcium requirements of older adults by including 3-4 daily serves of dairy foods (milk, cheese and yoghurt) (MedDairy). In our second trial $(\mathrm{n}=33$ ), we tested a Mediterranean diet supplemented with 2-3 weekly serves of fresh, lean pork (MedPork), designed to provide an alternate source of protein. Both trials employed a low-fat control diet (LF) and a 24-week parallel crossover design, consisting of two 8-week intervention periods and an 8-week washout separating interventions. We found that the MedDairy intervention significantly increased dairy food (mean difference $=1.0 \pm 0.2$ serves, $\mathrm{P}<0.001)$ and calcium intake (mean difference $=25.9 \pm 6.8 \mathrm{mg} / \mathrm{MJ}, \mathrm{P}<0.001)$ compared to LF. Further, MedDairy led to greater improvements in morning home systolic blood pressure (mean difference $=-1.6 \pm$ $0.6 \mathrm{mmHg}, \mathrm{P}=0.01$ ), triglycerides (mean difference $=-0.05 \pm 0.02 \mathrm{mmol} / \mathrm{L}, \mathrm{P}<0.01$ ), HDL (mean difference $=0.04 \pm 0.01 \mathrm{mmol} / \mathrm{L}$, $\mathrm{P}=<0.01$ ), and total cholesterol to HDL ratio (mean difference $=-0.4 \pm 0.10 \mathrm{mmol} / \mathrm{L}, \mathrm{P}=<0.001$ ). The MedDairy intervention also led to greater improvements in processing speed $(\mathrm{P}=0.04)$, a measure of cognitive function, as well as self-reported mood $(\mathrm{P}$ $=0.01)$. No significant differences were observed between MedPork and LF for blood pressure or other markers of cardiometabolic health. However, the MedPork intervention led to greater improvements in processing speed $(P=0.01)$ and $\operatorname{mood}(P=0.03)$. Our findings demonstrate that a Mediterranean diet with added dairy foods is capable of delivering adequate calcium to ageing populations while providing cardiovascular and cognitive benefits. Further, a Mediterranean diet supplemented with fresh, lean pork offers comparable cardiovascular benefits to a low-fat diet and greater improvements to cognitive function and mood. Our findings are of particular relevance to non-Mediterranean populations at risk of cardiovascular disease and dementia, and offer two options for modifying the Mediterranean diet depending on dietary priorities.

\section{Conflict of Interest}

There is no conflict of interest. 